PHYSICA SCRIPTA

(The Royal Swedish Academy of Sciences)

Online ISSN: 1402-4896; Print ISSN: 0031-8949; Impact factor = 1.902

Publisher- Institute of Physics (IOP)

Accepted May $8^{\text {th }} 2019$

\title{
METACHRONAL PROPULSION OF A MAGNETIZED PARTICLE-FLUID SUSPENSION IN A CILIATED CHANNEL WITH HEAT AND MASS TRANSFER
}

\author{
Sara I. Abdelsalam ${ }^{1,2}$, M. M. Bhatti ${ }^{3, *}$, A. Zeeshan ${ }^{4}$, A. Riaz ${ }^{5}$, and O. Anwar Bég6 \\ ${ }^{1}$ Basic Science Department, Faculty of Engineering, The British University in Egypt, \\ Al-Shorouk City, Cairo 11837, Egypt. \\ ${ }_{2}^{2}$ Instituto de Matemáticas - Juriquilla, Universidad Nacional Autónoma de México, Blvd. Juriquilla 3001, \\ Querétaro, 76230, México. \\ ${ }^{3}$ Shanghai Institute of Applied Mathematics and Mechanics, Shanghai University, Shanghai China. \\ ${ }^{4}$ Department of Mathematics \& Statistics, FBAS, International Islamic University Islamabad, Pakistan. \\ ${ }^{5}$ Department of Mathematics, University of Education Lahore, Jauharabad Campus, Jauharabad, Pakistan. \\ ${ }^{6}$ Multi-Physical Engineering Sciences, Mechanical/Aeronautical Engineering, University of Salford, M54WT, \\ United Kingdom. \\ *Email: muhammad09@shu.edu.cn; mubashirme@yahoo.com
}

\section{ABSTRACT:}

Biologically inspired pumping systems are of great interest in modern engineering since they achieve enhanced efficiency and circumvent the need for moving parts and maintenance. Industrial applications also often feature two-phase flows. In this article, motivated by these applications, the pumping of an electrically conducting particle-fluid suspension due to metachronal wave propulsion of beating cilia in a two-dimensional channel with heat and mass transfer under a transverse magnetic field is investigated theoretically. The governing equations for mass and momentum conservation for fluid- and particle-phases are formulated by ignoring the inertial forces and invoking the long wavelength approximation. The Jeffrey viscoelastic model is employed to simulate non-Newtonian characteristics. The normalized resulting differential equations are solved analytically. Symbolic software is employed to evaluate the results and simulate the influence of different parameters on flow characteristics. Results are visualized graphically with carefully selected and viable data. With increasing wave number $(\beta)$ fluid velocity is accelerated in the core region whereas it is decelerated near the channel wall, for the Newtonian case. With increasing eccentricity of cilia elliptic path $(\alpha)$, a similar response is computed as for the wave number. The size of the bolus is enhanced (and quantity of boluses is reduced) with increasing eccentricity of the cilia elliptic path $(\alpha)$ and Hartmann (magnetic) number $(M)$ whereas bolus size is decreased (and quantity of boluses is increased) with increasing wave number $(\beta)$ and particle volume fraction $(C)$. It is also noted that increasing Schmidt number $(S c)$ and Soret number $(\mathrm{Sr})$ diminish the concentration magnitudes. Furthermore, Brinkman number (which represents viscous heating effects) significantly boosts the temperature magnitudes. The current analysis provides a useful benchmark for more general computational simulations.

KEYWORDS: Metachronal wave; magnetohydrodynamics; particle-fluid suspension; Cilia 
motion; bio-inspired pumping; non-Newtonian model.

\section{INTRODUCTION}

The word cilia is derived from the Latin for "eyelashes". They arise in many biological applications both externally and internally (e.g. eukaryotic cells) and assist in generating bending waves which transport fluid over complex surfaces. Each cilium has a range length of about 2 micrometers to millimetres and its diameter is 0.2 micrometers. The term cilium is used when the cellular appendages are smaller in size and bound together on a single cell. The inner structure of cilia is characterized by a cylindrical core called an axoneme. Within the cilia there exists an arrangement of force-generating molecular motors known as dynein and elastic filaments which are termed microtubules. Cilia elements are alike to hair-like motile appendages which are found in female and male reproductive tracts, digestive systems and in the nervous system. Cilia motion plays a significant role in different physiological processes i.e. circulation, reproduction, respiration, alimentation and locomotion. When cilia elements conglomerate, a large-scale motion of propagating waves occurs, and this process is known as a metachronal wave. On protozoa (ciliated surfaces) when the beating of cilia occurs in large amounts, the activity of different cilia contributes collectively to the hydrodynamics and generates metachronal waves. Various authors [1-4] investigated the fluid dynamics associated with metachronal wave cilia beating and observed that the fluid viscosity changes across the thickness of physiological organs such as the duct afferents of the male reproductive tract, cervical canal and intestines. The behaviour and structure of a typical cilium can be treated as a low Reynolds number swimmer, owing to the properties of displacing and pushing a fluid with an impact of a total force. Cilia motion is generally associated with asymmetric beating which comprises two different phases, namely the recovery stroke and the effective stroke. Agrawal and Anwaruddin [5] investigated the cilia transport of Newtonian fluid having variable viscosity. However, the vast majority of biological fluent media are nonNewtonian in nature and therefore more accurate modelling of ciliated propulsion requires rheological models which may be viscoplastic, viscoelastic, micro-structural etc. Recently, Nadeem and Sadaf [6] presented closed-form solutions for nanofluid propulsion via cilia motion. Akbar and Butt [7] analysed the metachronal wave propulsion of a Rabinowitsch fluid in the presence of heat transfer. Miltran [8] conducted a three-dimensional formulation of metachronal waves in rows of pulmonary cilia using a two-layer fluid model (Newtonian viscous fluid adjacent to the cilia bases and viscoelastic fluid in which the tips of the cilia move) using an immersed 
boundary method. Maqbool et al. [9] analysed the metachronal propulsion of fractional generalized Burgers fluid in a titled conduit. Siddiqui et al. [10] employed a power-law Ostwald DeWaele model for flow in a ciliated channel. Akbar et al. [11] used Eringen's micropolar model to derive closed-form solutions for ciliated propulsion in a two-dimensional channel.

Magnetohydrodynamics (MHD) is also an active area of modern biomedical engineering sciences. Blood for example is electrically conducting owing to the presence of haemoglobin in the iron molecule and ions [12]. Other physiological fluids which respond to magnetic fields are synovial lubricants [13] and plasma [14]. To properly quantify the effectiveness of, for example, magnetic drug targeting for different cancerous diseases $[15,16]$, it is important to develop realistic magnetohydrodynamic physiological flow models [17]. The imposition of external (extracorporeal) magnetic fields is also beneficial in pain therapy [18] since it successfully controls flow. Furthermore, smart electromagnetic medical micro-pump design (e.g. non-pulsating) combines ciliated channel features with magnetohydrodynamics $[19,20]$. The relative contribution of viscous hydrodynamic force and Lorentz magnetic drag force must be carefully selected in such designs. In recent years, significant interest has been directed towards simulating internal magnetohydrodynamic biological propulsion flows in channels and tubes with non-Newtonian models. Manzoor et al. [21] used the Jeffrey viscoelastic model and the Adomian decomposition method to investigate hydromagnetic ciliated flow and heat transfer in a porous medium channel with viscous dissipation effects. They used an elliptic model for cilia beating and showed that pressure difference is enhanced with increasing permeability \& Jeffery first parameter ("relaxation to retardation time ratio") whereas it is reduced with Hartmann (magnetic) numbers. Akbar et al. [22] used the Casson viscoplastic model to analyse magnetic flow and convection in a ciliated channel with wall slip effects under an inclined magnetic field.

Although physiological fluids are known to be non-Newtonian they have the further complication that they contain multiple phases. Blood for example contains numerous suspensions which include non-protein hormones, lipids, proteins, nutrients, electrolytes, gases, erythrocytes, leukocytes etc. To accurately simulate the multi-phase nature of blood more sophisticated models are required. Among the most amenable is the fluid-particle suspension theory [23] which analyses the fluent medium with separate conservation equations for the fluid phase and particulate (suspension) phases. Also known as the "dusty fluid model", this approach is also applicable to magnetohydrodynamic pumps (which may contain metallic particles e.g. seeded potassium), 
aerosols, slurry systems, petroleum and centrifugal separation processes, particulate deposition on semi-conductor wafers electrostatic precipitation. The dusty model was introduced by Saffman [24] who considered hydrodynamic stability of fluid-particle suspension for a gas. Marble [25] generalized the model and identified important new applications in mechanical engineering sciences. Zung [26] conducted an early analysis of swirling Von Karman flows of fluid-particle suspensions. The continuum fluid-particle theory is also applicable in various biological systems of hydrodynamics such as swimming of microorganisms, rheology of blood, diffusion of proteins and particle deposition. Interesting applications of fluid-particle suspension theory in medical engineering include Mekheimer et al. [27, 28] and Srivastava and Srivastava [29] for peristaltic flows, Bhatti et al. [30] and Kamel et al. [31] for endoscopic slip flows, Chakraborty et al. [32] for stenotic hemodynamics, Bég et al. [33-35] for hematological filtration flows (dialysis). Magnetohydrodynamic dusty flows have also garnered some attention in recent years. Relevant studies include Vajravelu and Nayfeh [36] (on stretching sheets), Hatami et al. [37] (who used a differential quadrature method for fluid-particle Coutte flow), Mahanthesh et al. [38] (on rotating magnetic lubrication) and Ramesh et al. [39] (on Hall peristaltic rheological hydromagnetic blood micropumps).

Moreover, the simulation of particle-fluid dynamics with heat and mass transfer has many scientific and engineering applications. These include thermal insulation, enhanced oil recovery, transport of underground energy, cooling of nuclear reactors, packed bed catalytic reactors vasodilation, haemo-dialysis process, oxygenation, treatment of hyperthermia and heat convection due to blood flow in a living body from the pores of tissues. Some relevant studies of fluid-particle transport phenomena include Refs. [40-45].

Motivated by recent developments in bio-inspired magnetic cilia systems [46], the aim of the present investigation is to analyze the magnetohydrodynamic pumping of a fluid-particle suspension due to metachronal wave propulsion of beating cilia with the viscoelastic Jeffrey fluid model. This non-Newtonian model represents biophysical fluids reasonably well and features three constants i.e. viscosity at zero shear rate, and two time-related material parameter constants. A number of studies have reported on the suitability of the Jeffery rheological model for biological hydrodynamics including Maraj et al. [47], Tripathi et al. [48] and Ellahi et al. [49]. However, to the authors' knowledge the collective fluid-particle and Jefferys viscoelastic models have not been considered simultaneously in ciliated magnetohydrodynamics. This constitutes the novelty of the 
present analysis. The governing mass and momentum conservation equations of motion for fluid phase and particulate phase are constructed under the assumption of long wave length and low Reynolds number approximation. The appropriate stress tensor terms for the Jeffery elasticviscous model are incorporated as are the Lorentzian magnetic body force terms. An elliptic beating cilia model is adopted to simulate metachronal wave propulsion [50]. The non-dimensional emerging ordinary differential equation boundary value problem is then solved analytically subject to appropriate boundary conditions. A detailed parametric study of the influence of wave number, cilia path eccentricity, magnetic Hartmann number and particle volume fraction on velocity, pressure and bolus characteristics is conducted with extensive visualization. Elaborate interpretation of the physics of the flow is included.

\section{MATHEMATICAL MODEL}

The physical regime under consideration is illustrated in Fig. 1. Unsteady hydromagnetic flow of an incompressible electro-conductive viscoelastic fluid-particle suspension through a ciliated twodimensional planar channel. The particles embedded in the fluid are assumed to be equal in size, spherical in shape, and uniformly distributed in a fluid. The volume fraction and interparticle collision of the particles are ignored. Stokes' linear drag theory is applied to model the drag force. An extrinsic magnetic field is applied, while the induced magnetic field is very small and assumed to be ignored here. Electrostatic interactions between the particles are ignored. A metachronal wave occurs due to collective beating of cilia along the walls and travels with a constant velocity $\tilde{c}$. A Cartesian coordinate system is adopted for the channel i.e. $\tilde{X}$-axis lie across the axial direction and $\tilde{Y}$-axis lie along the transverse direction (see Fig. 1). The envelop for cilia tips is supposed to follow an elliptic model and the appropriate equations [22] are:

$$
\begin{aligned}
& \bar{F}(\tilde{t}, \tilde{X})=\tilde{a}+\varepsilon \tilde{a} \cos \frac{2 \pi}{\lambda}(\tilde{X}-\tilde{c} \tilde{t}), \\
& \bar{G}(\tilde{t}, \tilde{X})=\tilde{X}_{0}+\varepsilon \tilde{a} \alpha \sin \frac{2 \pi}{\lambda}(\tilde{X}-\tilde{c} \tilde{t}) .
\end{aligned}
$$




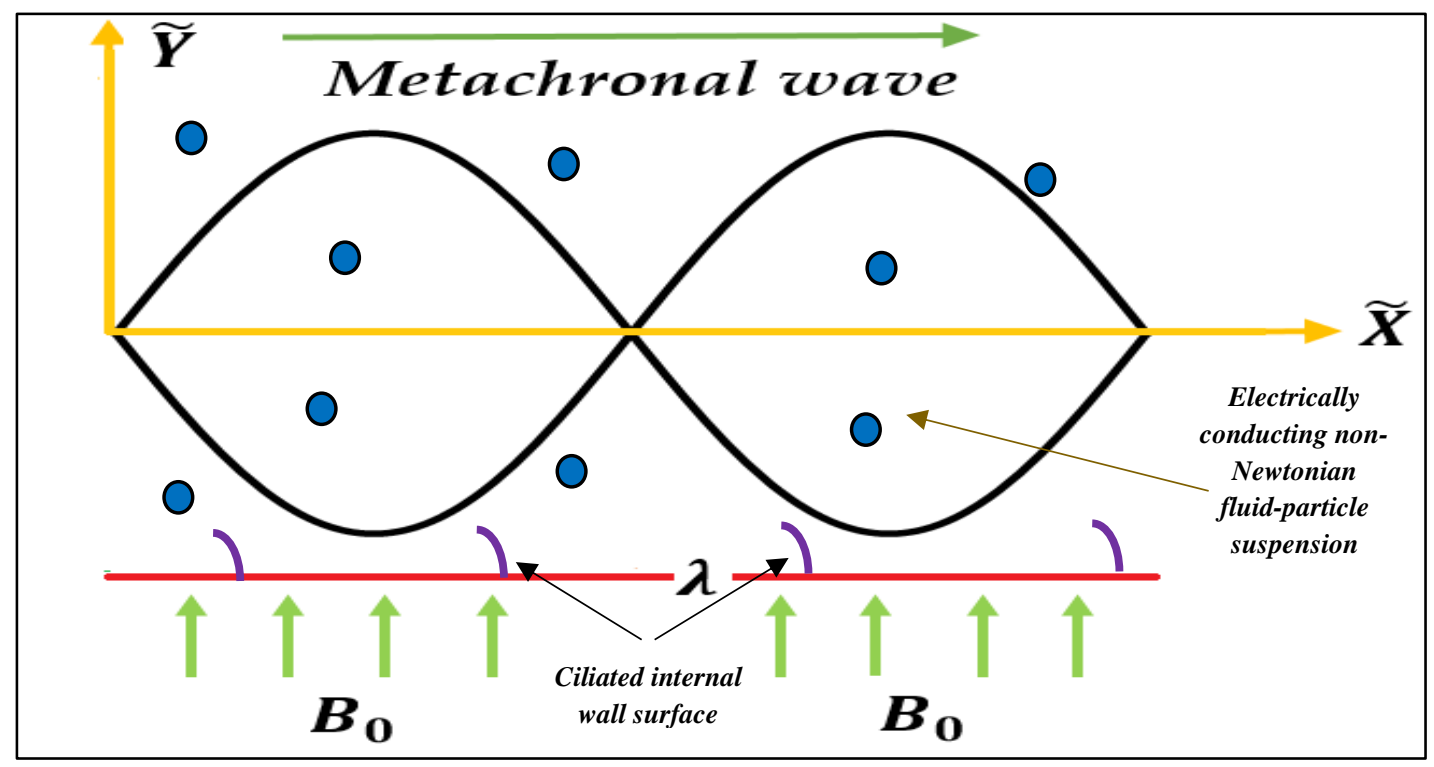

Fig. 1: Flow configuration for metachronal magnetic pumping.

The horizontal and vertical velocity components for the cilia motion read as [22]:

$$
\begin{gathered}
\tilde{U}_{f, p}=\frac{-\frac{2 \pi}{\lambda} \tilde{a} \varepsilon \alpha \tilde{c} \cos \frac{2 \pi}{\lambda}(\tilde{X}-\tilde{c} \tilde{t})}{1-\frac{2 \pi}{\lambda} \tilde{a} \varepsilon \alpha \tilde{c} \cos \frac{2 \pi}{\lambda}(\tilde{X}-\tilde{c} \tilde{t})}, \\
\tilde{V}_{f, p}=\frac{-\frac{2 \pi}{\lambda} \tilde{a} \varepsilon \alpha \tilde{c} \sin \frac{2 \pi}{\lambda}(\tilde{X}-\tilde{c} \tilde{t})}{1-2 \frac{2 \pi}{\lambda} \tilde{a} \varepsilon \alpha \tilde{c} \sin \frac{2 \pi}{\lambda}(\tilde{X}-\tilde{c} \tilde{t})} .
\end{gathered}
$$

The governing equations of motion for the fluid- and particulate-phases read as:

\section{Fluid Phase [51-52]:}

$$
\begin{aligned}
& -\frac{\partial \tilde{V}_{f}}{\partial \tilde{Y}}=\frac{\partial \tilde{U}_{f}}{\partial \tilde{X}}, \\
& \left(\tilde{U}_{f} \frac{\partial \tilde{U}_{f}}{\partial \tilde{X}} \rho_{f}+\rho_{f} \frac{\partial \tilde{U}_{f}}{\partial \tilde{t}}+\rho_{f} \tilde{V}_{f} \frac{\partial \tilde{U}_{f}}{\partial \tilde{Y}}\right)=\frac{\partial}{\partial \tilde{X}} \mathrm{~S}_{\tilde{X} \tilde{X}}+\frac{\partial}{\partial \tilde{Y}} \mathrm{~S}_{\tilde{X} \tilde{Y}}-\frac{\partial \tilde{P}}{\partial \tilde{X}}+\frac{C S\left(\tilde{U}_{p}-\tilde{U}_{f}\right)}{(1-C)}-\frac{\sigma B_{0}^{2} \tilde{U}}{(1-C)} \\
& \left(\tilde{V}_{f} \frac{\partial \tilde{V}_{f}}{\partial \tilde{Y}}+\tilde{U}_{f} \frac{\partial \tilde{V}_{f}}{\partial \tilde{X}}+\frac{\partial \tilde{V}_{f}}{\partial \tilde{t}}\right) \rho_{f}=\frac{\partial}{\partial \tilde{Y}} \mathrm{~S}_{\tilde{Y} \tilde{Y}}+\frac{\partial}{\partial \tilde{X}} \mathrm{~S}_{\tilde{X} \tilde{Y}}+\frac{C S\left(\tilde{V}_{p}-\tilde{V}_{f}\right)}{(1-C)}-\frac{\partial \tilde{P}}{\partial \tilde{Y}}
\end{aligned}
$$




$$
\begin{aligned}
& \left(c_{p}-c_{p} C\right) \rho_{f}\left(\tilde{V}_{f} \frac{\partial \vartheta_{f}}{\partial \tilde{Y}}+\tilde{U}_{f} \frac{\partial \vartheta_{f}}{\partial \tilde{X}}+\frac{\partial \vartheta_{f}}{\partial \tilde{t}}\right)-\frac{\rho_{p} c_{p} C}{\varpi_{T}}\left(\vartheta_{p}-\vartheta_{f}\right)= \\
& (1-C) \mathbf{S}_{\tilde{X} \tilde{X}}\left(\frac{\partial \tilde{U}_{f}}{\partial \tilde{Y}}\right)+\mathrm{k}(1-C)\left(\frac{\partial^{2} \vartheta_{f}}{\partial \tilde{X}^{2}}+\frac{\partial^{2} \vartheta_{f}}{\partial \tilde{Y}^{2}}\right)+C S\left(\tilde{U}_{f}-\tilde{U}_{p}\right)^{2}, \\
& \left(\tilde{U}_{f} \frac{\partial \Theta_{f}}{\partial \tilde{X}}+\frac{\partial \Theta_{f}}{\partial \tilde{t}}+\tilde{V}_{f} \frac{\partial \Theta_{f}}{\partial \tilde{Y}}\right)(1-C)=(1-C) \mathrm{D}_{\mathrm{m}}\left(\frac{\partial^{2} \Theta_{f}}{\partial \tilde{Y}^{2}}+\frac{\partial^{2} \Theta_{f}}{\partial \tilde{X}^{2}}\right) \\
& +\frac{\rho_{p} C}{\rho_{f} \varpi_{C}}\left(\Theta_{p}-\Theta_{f}\right)+\frac{\mathrm{D}_{\mathrm{m}}}{T_{m}}\left(\frac{\partial^{2} \Theta_{f}}{\partial \tilde{X}^{2}}+\frac{\partial^{2} \Theta_{f}}{\partial \tilde{Y}^{2}}\right)(1-C) \mathrm{K}_{T} .
\end{aligned}
$$

\section{Particulate Phase [51-52]:}

$$
\begin{aligned}
& \frac{\partial \tilde{U}_{p}}{\partial \tilde{X}}+\frac{\partial \tilde{V}_{p}}{\partial \tilde{Y}}=0, \\
& C\left(\tilde{U}_{p} \frac{\partial \tilde{U}_{p}}{\partial \tilde{X}}+\frac{\partial \tilde{U}_{p}}{\partial \tilde{t}}+\tilde{V}_{p} \frac{\partial \tilde{U}_{p}}{\partial \tilde{Y}}\right) \rho_{p}=-\frac{\partial \tilde{P}}{\partial \tilde{X}} C+C\left(\tilde{U}_{f}-\tilde{U}_{p}\right) S, \\
& C\left(\tilde{U}_{p} \frac{\partial \tilde{V}_{p}}{\partial \tilde{X}^{\prime}}+\frac{\partial \tilde{V}_{p}}{\partial \tilde{t}}+\tilde{V}_{p} \frac{\partial \tilde{V}_{p}}{\partial \tilde{Y}}\right) \rho_{p}=-C \frac{\partial \tilde{P}}{\partial \tilde{Y}}+S\left(\tilde{V}_{f}-\tilde{V}_{p}\right) C \\
& C c_{p} \rho_{p}\left(\tilde{V}_{p} \frac{\partial \vartheta_{p}}{\partial \tilde{Y}}+\tilde{U}_{p} \frac{\partial \vartheta_{p}}{\partial \tilde{X}}+\frac{\partial \vartheta_{p}}{\partial \tilde{t}}\right)=\frac{\rho_{p} C c_{p}}{\varpi_{T}}\left(\vartheta_{f}-\vartheta_{p}\right), \\
& \left(\frac{\partial \Theta}{\partial \tilde{t}}+\tilde{V}_{p} \frac{\partial \Theta}{\partial \tilde{Y}}+\tilde{U}_{p} \frac{\partial \Theta_{p}}{\partial \tilde{X}}\right)=\left(\frac{\Theta_{f}-\Theta_{p}}{\varpi_{C}}\right) .
\end{aligned}
$$

The empirical relations for the drag coefficient $(S)$ and the viscosity of suspension $\left(\mu_{\mathrm{s}}\right)$ are given as follows [39]:

$$
S=\frac{9 \mu_{0}}{2 \hat{a}^{2}} \tilde{\lambda}(C), \tilde{\lambda}(C)=\frac{4+3(C+\sqrt{[8-3 C] C})}{(3 C-2)^{2}}, \mu_{s}=\frac{\mu_{0}}{1-\chi C}, \chi=0.07 e^{\frac{1}{T}\left[2.49 T C+1107 e^{-1.69 C}\right]} .
$$

In Eqns. (1)-(11) all parameters are defined in the notation at the start of the paper. The stress tensor in the Jeffrey fluid model is:

$$
\mathbf{S}=\frac{\mu_{s}}{1+\lambda_{1}}\left(\dot{\gamma}+\lambda_{2} \ddot{\gamma}\right)
$$

Here $\mu_{s}$ is dynamic viscosity of Jefferys fluid, $\dot{\gamma}$ rate of strain, $\lambda_{1}$ ratio of relaxation and retardation time (Jeffreys parameter), $\lambda_{2}$ retardation time while dots denote differentiation with respect to time. Eqn. (12) can be reduced to the Newtonian case by taking $\lambda_{1}=0$. It is convenient to 
transform variables from the fixed frame to the laboratory (wave) frame which maps the flow into a moving boundary problem and eliminates time:

$$
\tilde{v}_{f, p}=\tilde{V}_{f, p}, \tilde{x}=\tilde{X}-\tilde{c} \tilde{t}, \tilde{U}_{f, p}-\tilde{u}_{f, p}=\tilde{c}, \tilde{P}=\tilde{p}, \tilde{y}=\tilde{Y}, .
$$

The non-dimensional quantities are defined as

$$
\begin{aligned}
& N=\frac{S \tilde{a}^{2}}{\mu_{s}}, M=\sqrt{\frac{B_{0}^{2} \tilde{a}^{2} \sigma}{\mu_{s}}}, S_{c}=\frac{\mu_{s}}{\rho \mathrm{D}_{\mathrm{m}}}, S_{r}=\frac{\rho \mathrm{D}_{\mathrm{m}} \mathrm{K}_{T}}{\mu_{s} T_{\mathrm{m}}}\left(\frac{\vartheta_{1}-\vartheta_{0}}{\Theta_{1}-\Theta_{0}}\right), B_{r}=P_{r} E_{c}, \\
& \left.\begin{array}{l}
x=\frac{\tilde{x}}{\lambda}, y \tilde{a}=\tilde{y}, v_{f, p}=\frac{\tilde{v}_{f, p}}{\tilde{c} \delta}, p=\frac{1}{\lambda \tilde{c} \mu_{s}} \tilde{p} \tilde{a}^{2}, \operatorname{Re}=\frac{\rho \tilde{a} \tilde{c}}{\mu_{s}}, \tilde{c} u_{f, p}=\tilde{u}_{f, p}, P_{r}=\frac{\mu_{s} c}{\mathrm{k}} \\
\Phi_{f, p}=\left(\Theta_{f, p}-\Theta_{0}\right)\left(\Theta_{1}-\Theta_{0}\right)^{-1}, \theta_{f, p}=\left(\vartheta_{f, p}-\vartheta_{0}\right)\left(\vartheta_{1}-\vartheta_{0}\right)^{-1}, E_{c}=\frac{\tilde{c}^{2}}{c_{p}\left(\vartheta_{1}-\vartheta_{0}\right)} .
\end{array}\right\},
\end{aligned}
$$

Here again all parameters are given in the notation. Using Eqn. (13) and Eqn. (14) in Eqns. (5) to (10), then employing the approximation of long wavelength and ignoring the inertial forces, leads to the following formulation.

The remaining equation for the fluid-phase read as:

$$
\begin{aligned}
& \frac{d p}{d x}=\frac{1}{\ell} \frac{\partial^{2} u_{f}}{\partial y^{2}}-M^{2}\left(u_{f}+1\right)+\frac{N C}{(1-C)}\left(u_{p}-u_{f}\right), \\
& \frac{\partial^{2} \theta_{f}}{\partial y^{2}}+P_{r} E_{c} \frac{1}{\ell}\left(\frac{\partial u_{f}}{\partial y}\right)^{2}=-\frac{P_{r} E_{c}}{N(1-C)}\left(\frac{d p}{d x}\right)^{2}, \\
& \frac{1}{S_{c}} \frac{\partial^{2} \Phi_{f}}{\partial y^{2}}=-S_{r} \frac{\partial^{2} \theta_{f}}{\partial y^{2}} .
\end{aligned}
$$

Similarly, for the particulate phase:

$$
\begin{aligned}
& \frac{d p}{d x}=N\left(u_{f}-u_{p}\right), \\
& \theta_{f}=\theta_{p}, \\
& \Phi_{f}=\Phi_{p} .
\end{aligned}
$$

Their corresponding boundary conditions are

$$
\begin{aligned}
& u_{f}^{\prime}=0 \text { at } y=0 ; u_{f}=-\frac{2 \phi \pi \cos 2 \pi(x) \beta \alpha}{-2 \phi \pi \cos 2 \pi(x) \beta \alpha+1}-1 ; \text { at } y=h=\phi \cos 2 \pi x+1, \\
& \theta_{f}=0=\Phi_{f} ; \text { at } y=0 \text { and } \theta_{f}=1=\Phi_{f} ; \text { at } y=h .
\end{aligned}
$$


where $\ell=1+\lambda_{1}$.

\section{ANALYTICAL SOLUTIONS}

The exact solutions of Eqn. (15) and Eq. (16) using Eqn. (17), can be obtained by integrating twice:

$$
\begin{aligned}
u_{f} & =-1-\frac{1}{(1-C) M^{2}} \frac{d p}{d x}-\left[(1-C) M^{2} N_{1}-\frac{d p}{d x}\right] \frac{\cosh [M y \sqrt{\ell}] \operatorname{sech}[h M \sqrt{\ell}]}{(-1+C) M^{2}}, \\
u_{p} & =u_{f}-\frac{1}{N} \frac{d p}{d x}, \\
\theta_{f, p} & =\left[4+\left(2 \theta_{1}-\theta_{2} \theta_{3}^{2}\right) h(h-y)\right] \frac{y}{h}+\frac{\theta_{2} \theta_{3}^{2}[h-y+y \cosh [2 h M \sqrt{\ell}]-h \cosh [2 y M \sqrt{\ell}]]}{8 h M^{2} \ell}, \\
\Phi_{f, p} & =\left[4-\left(2 \theta_{1}-\theta_{2} \theta_{3}^{2}\right) h S_{r} S_{c}(h-y)\right] \frac{y}{h}+\frac{S_{r} \theta_{2} \theta_{3}^{2} S_{c}[y-h-y \cosh [2 h M \sqrt{\ell}]+h \cosh [2 y M \sqrt{\ell}]]}{8 h M^{2} \ell} .
\end{aligned}
$$

Where

$$
\begin{aligned}
& N_{1}=\frac{2 \pi \phi \alpha \beta \cos 2 \pi(x)}{1-2 \pi \phi \alpha \beta \cos 2 \pi(x)}, \\
& \theta_{1}=\frac{B_{r}}{N_{1}(1-C)}\left(\frac{d p}{d x}\right)^{2}, \\
& \theta_{2}=\frac{B_{r}}{\left(1-\lambda_{1}\right)}, \\
& \theta_{3}=-\left[M^{2}\left(N_{1}-C N_{1}\right)-\frac{d p}{d x}\right] \frac{\sqrt{\ell}(\operatorname{sech}[h M \sqrt{\ell}])}{(1-C) M},
\end{aligned}
$$

Volumetric flow rate can be obtained by integration across the channel width:

$$
\begin{aligned}
& Q_{f}=-(C-1) \int_{0}^{h} u_{f} \mathrm{dy}, \\
& Q_{p}=C \int_{0}^{h} u_{p} \mathrm{dy},
\end{aligned}
$$

where

$$
Q=Q_{f}+Q_{p}
$$

And

$$
Q=\frac{h \sqrt{\ell} M\left[\frac{d p}{d x}+(1-C) M^{2}\left(1+\frac{C}{N} \frac{d p}{d x}\right)\right]+\left(\left(M^{2}-M^{2} C\right) N_{1}-\frac{d p}{d x}\right) \tanh [h M \sqrt{\ell}]}{\sqrt{\ell}\left(-M^{3}+M^{3} C\right)} .
$$


The non-dimensional pressure rise $\Delta p$ is solved numerically by:

$$
\Delta p=\int_{0}^{1} \hbar \mathrm{dx} .
$$

where pressure gradient $\hbar(=d p / d x)$ is evaluated from Eq. (38).

\section{GRAPHICAL DISCUSSION}

This section illustrates the graphical results for the impact of selected physical parameters on velocity profile and pressure rise. Effects of velocity are plotted in Fig. (2) and Fig. (3), while the pumping characteristics are sketched in Fig. (4) and Fig. (5) and Streamlines are also drawn in Fig. (6) to Fig. (9) for selected parameters. Fig. (10) to Fig. (14) are plotted for concentration and temperature profiles.

Fig. (2) indicates that when cilia eccentricity parameter $(\alpha)$ increases there is a deceleration in the fluid phase velocity $\left(u_{f}\right)$. In all cases the profiles decay monotonically from the channel centreline to the wall (no slip condition). Fig. 2 also shows that with increasing wave number, $(\beta)$, fluid velocity is accelerated in the core region whereas it is decelerated near the channel wall. With increasing eccentricity of cilia elliptic path, $(\alpha)$, therefore a similar response is computed as for the wave number. Fig. (3) shows that when the particle volume fraction rises then the velocity of the fluid decreases i.e. greater concentration of suspended particles induces a deceleration. It also indicates that the velocity of the fluid diminishes when the magnetic parameter $(M)$ rises. Higher values of $(M)$ correspond to stronger external magnetic field. This in turn accentuates the Lorentzian drag force which increases the impedance to the flow i.e. induces retardation and a reduction in fluid phase velocity magnitudes. At higher values of $y$, negative velocity is induced i.e. near the walls of the channel there is flow reversal. Evidently the flow is strongly regulated by the action of a magnetic field and this is of significance in flow control in micro-biofluidics.

Figs. (4) and (5) are plotted to visualize the impact of selected parameters on the ciliated channel pumping features. The nature of the flow is periodic and in fact a peristaltic wave. It can be observed from Fig. (4) that when cilia eccentricity parameter $(\alpha)$ increases then pressure rise diminishes in the retrograde pumping region $(Q<0, \Delta p>0)$ and its behaviour is similar in free 
pumping region $(Q<0, \Delta p<0)$ and also the co-pumping region $(Q>0, \Delta p<0)$. The behaviour of the amplitude ratio $(\phi)$ is found to be opposite in both the regions. Fig. (5) reveals that when the particle volume fraction $(C)$ rises then the pressure rise diminish in a retrograde pumping region, whereas opposite response is computed in the co-pumping region. Inspection of Fig. (5) also reveals that when the Hartmann number $(M)$ rises then the pressure rise reduces in the free pumping region and in co-pumping region whereas it distinctly increases in the retrograde pumping region. The magnetic field therefore modifies pressure distribution substantially in different regions of the pumping in the ciliated channel.

In peristaltic flows, a key mechanism of interest is trapping which may be analysed by drawing stream lines. Trapping refers to the formation of an internally circulating bolus that is enclosed by various stream lines. It can be seen from Fig. (6) that when cilia eccentricity parameter $(\alpha)$ rises then the magnitude of the bolus decreases whereas the number of boluses increases. From Fig. (7), we can see that the size of the trapping bolus decreases when the wave number $(\beta)$ increases. Fig. (8) demonstrates that when the particle volume fraction $(C)$ rises then the number of boluses increases whereas the size of the bolus decreases. Finally Fig. (9) indicates that when the Hartmann number $(M)$ rises from 0.5 through 1 to 1.5 , then the size of the bolus becomes bigger while the number of bolus reduces i.e. greater magnetic field encourages growth of the bolus whereas it inhibits the quantity of boluses formed in the channel. When the viscous force is exceeded by the Lorentz magnetic body force therefore (i.e. $M>1$ ) the maximum contraction in bolus size is achieved in the regime. The opposite effect is induced when the viscous force exceeds the magnetic body force $(M<1)$. The intermediate case $(M=1)$ corresponds to an equivalence in the viscous hydrodynamic force and the Lorentz magnetic drag force and produces an intermediate bolus magnitude.

Fig. (10) to Fig. (14) show the variation of temperature profiles and concentration profiles for both the fluid- and particulate-phase. From Fig. (10) can be observed from this figure that an enhancement in $\alpha$ and $B_{r}$ tends to rise the temperature profile. Brinkman number $B_{r}$ represents relative impact of heat generated due to viscous dissipation and the propagation of heat by molecular conduction. Therefore, an increment in Brinkman number $B_{r}$ tends to create less effect 
in the generation of thermal conduction through viscous dissipation and higher elevation in temperature profile. A similar behaviour was noted by Gorla et al. [53]. Fig. (11) is sketched for $\beta$ and $C$. It is noted from this figure that the particle volume fraction $C$ tends to produce a significant resistance in the temperature profile. However, the wavenumber $\beta$ reveals a similar behaviour and the temperature profile is maximum in the centre of the channel. Fig. (12) is plotted for $\alpha$ and $S_{r}$ (Soret number) for the concentration profile. It can be viewed from this figure that both parameters represent converse behaviour on the concentration profile. Soret number is a mechanism noticed in the mixtures of mobile particles where the multiple particles types reveal various responses to a force of the temperature gradient. Therefore, an enhancement in Soret number tends to reduce the concentration profile. In Fig. (13) we can observe that concentration profile rises due to significant influence of $\beta$ and $C$. However, the magnitude of the concentration profile is minimum in the centre of the channel. Fig. (14) shows the variation of $S_{c}$ (Schmidt number) and $B_{r}$ on the concentration profile. It can be seen from this figure that an enhancement in Schmidt number diminishes the concentration profile. Schmidt number represents the ratio of mass diffusivity and kinematic viscosity ("momentum diffusivity"), therefore, an enhancement in Schmidt number tends to rise the viscous diffusion and as a result concertation profile decreases.

\section{CONCLUSIONS}

A mathematical study has been conducted to simulate the transport of an electro-conductive viscoelastic fluid-particle suspension with heat and mass transfer via metachronal wave propulsion in a planar channel. The internal walls of the planar channel have been modelled as ciliated and the synchronized cilia beating generates the metachronal wave. The governing conservation equations for momentum and mass for both the fluid phase and particle phase have been normalized with appropriate dimensionless variables and simplified via lubrication approximations. The resulting ordinary differential boundary value problem has been solved analytically. Numerical evaluation of the fluid phase temperature, velocity, pressure rise, concentration and streamline distributions has been conducted with symbolic software (Mathematica). The trapping mechanism has been examined via drawing stream lines. Graphical results for non-Newtonian fluids have been visualized for the effect of cilia eccentricity parameter, 
Brinkman number, metachronal wave number, Schmidt number, particle volume fraction, Soret number and magnetic parameter. The major deductions which can be made from the present analysis are:

- Velocity of the fluid reveals opposite attitude along the walls with an increase in both the cilia eccentricity and wave number parameters.

- Velocity of the fluid diminish with increasing particle volume fraction and Hartman number.

- Pressure rise diminishes in the retrograde pumping due to an increment in particle volume fraction while the contrary attitude is observed in the co-pumping region.

- Temperature profile reveals converse behaviour for higher values of Brinkman number and particle volume fraction.

- Soret number and Schmidt number produces similar impact on the concentration profile.

The present results have ignored Hall current [39] and magnetic induction effects which may also be relevant to biological magnetic devices and blood flow control. These will be considered in the future. Furthermore nanofluids (featuring either metallic [54] or carbon-based [55] nano-particles) offer some potential in smart biomimetic pumping systems and these are also under consideration.

\section{ACKNOWLEDGEMENTS}

This work was accomplished under a bilateral cooperation agreement between TWAS-UNESCO and Universidad Nacional Autónoma de México in Querétaro, Juriquilla. Sara I. Abdelsalam would like to acknowledge TWAS-Italy for the financial support of her visit to UNAM under the TWAS-UNESCO Associateship. The author also thanks UNAM for the financial support under the aforementioned agreement. The authors are also grateful to both reviewers for their constructive comments which have served to improve the present manuscript.

\section{REFERENCES}

[1] Barton C, Raynor S, Analytical investigation of cilia induced mucous flow. Bulletin Mathematical biophysics. 29: 419-428 (1967).

[2] Blake JR, A spherical envelope approach to ciliary propulsion. J. Fluid Mech. 46: 199-208 (1971). 
[3] Guha SK, Kaur H, Ahmed AM, Mechanics of spermatic fluid transport in the vas deferens. Medical Biological Engineering, 13: 518-522 (1975).

[4] Peter S, Studies on cilia III. Further studies on the cilium tip and a "sliding filament" model of ciliary motility. Journal Cell Biology. 39: 77-94 (1968).

[5] Agarwal HL, Anawaruddin, Cilia transport of Bio-fluid with variable viscosity, Indian Journal Pure Applied Mathematics, 15: 1128-1139 (1984).

[6] Nadeem S, Sadaf H, Trapping study of nanofluids in an annulus with cilia. AIP Advances. 5: 127204 (2015).

[7] Akbar SN, Butt AW, Heat transfer analysis of Rabinowitsch fluid flow due to metachronal wave of cilia. Results in Physics. 5: 92-98 (2015).

[8] S.M. Mitran, Metachronal wave formation in a model of pulmonary cilia, Comp. Struc. 85(1114): 763-774 (2007).

[9] K. Maqbool, Mann, A.B., Siddiqui, A.M., Shaheen, S., Fractional generalized Burgers' fluid flow due to metachronal waves of cilia in an inclined tube. Adv. Mech. Eng., 9, 1687814017715565 (2017).

[10] Siddiqui A. M., Haroon T., Rani M., and Ansari A. R. (2010). An analysis of the flow of a power law fluid due to ciliary motion in an infinite channel, J. Biorheology, 24 (2) 56-69.

[11] N. S. Akbar, D. Tripathi, Z. H. Khan and O. Anwar Bég, Mathematical modelling of pressuredriven micropolar biological flow due to metachronal wave propulsion of beating cilia, Mathematical Biosciences, 301, 121-128 (2018).

[12] Cano, M. E., Gil-Villegas, A., Sosa, M. A., Villagómez, J. C., \& Baffa, O. (2006). Computer simulation of magnetic properties of human blood. Chemical physics letters, 432(4-6), 548552.

[13] Chernyakova, Y. M., Pinchuk, L. S., Lobanovskii, L. S., Drozd, E. S., \& Chizhik, S. A. (2011). Structure and magnetic susceptibility of lubricating fluids in synovial joints. Journal of Friction and Wear, 32(1), 54-60.

[14] Liu, C. H., Wu, W. C., Lai, H. Y., \& Hou, H. Y. (2011). Magnetic purification of plasminogen from human plasma by specific lysine affinity. Journal of bioscience and bioengineering, 112(3), 219-224.

[15] Voltairas PA, Fotiadis DI, Michalis LK. Hydrodynamics of magnetic drug targeting, $J$. Biomechanics. 35 (6):813-21 (2002). 
[16] Abdelsalam SI and Bhatti MM. New insight into AuNP applications in tumor treatment and cosmetics through wavy annuli at the nanoscale, Scientific Reports. 9 (1): 1-14 (2019).

[17] Abdelsalam SI and Bhatti MM. The impact of impinging TiO2 nanoparticles in Prandtl nanofluid along with endoscopic and variable magnetic field effects on peristaltic blood flow, Multidiscipline Modeling in Materials and Structures. 14 (3): 530-548 (2018).

[18] Shapiro B, Kulkarni S, Nacev A, Sarwar A, Preciado D, Depireux DA. Shaping magnetic fields to direct therapy to ears and eyes, Ann. Rev Biomed Eng., 16:455-81 (2014).

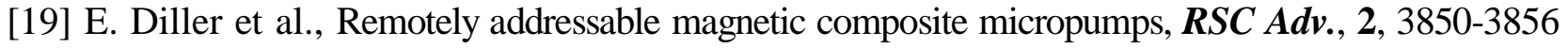
(2012).

[20] Y-N. Wang, Micropumps and biomedical applications - A review, Microelectronic Engineering, 195, 121-138 (2018).

[21] N. Manzoor, K. Maqbool, O. Anwar Bég and S. Shaheen, Adomian decomposition solution for propulsion of dissipative magnetic Jeffrey biofluid in a ciliated channel containing a porous medium with forced convection heat transfer, Heat Transfer - Asian Research, 1-26 (2018). DOI: $10.1002 / \mathrm{htj} .21394$

[22] N. S. Akbar, D. Tripathi, O. Anwar Bég, Z. H. Khan, MHD dissipative flow and heat transfer of Casson fluids due to metachronal wave propulsion of beating cilia with thermal and velocity slip effects under an oblique magnetic field, Acta Astronautica, 128, 1-12 (2016).

[23] V.P. Srivastava, Particle-fluid suspension model of blood flow through stenotic vessels with applications, International Journal of Bio-Medical Computing, 38, 141-154 (1995).

[24] P.G. Saffman, The stability of a laminar flow of a dusty gas, J. Fluid Mech, 13 (3) 120-128 (1962).

[25] F.E. Marble, Dynamics of dusty gases, Ann. Rev. Fluid Mech, 2, 397-446 (1970).

[26] L.B. Zung, Flow induced in fluid-particle suspension by infinite rotating disk, Phys. Fluids, 12, 18-23 (1969).

[27] Mekheimer KS, El Shehawey EF, Elaw AM, Peristaltic motion of a particle-fluid suspension in a planar channel. International Journal Theoretical Physics. 37: 2895 (1998).

[28] Mekheimer KS, Elmaboud YA, Peristaltic transport of a particle-fluid suspension through a uniform and non-uniform annulus. Applied Bionics and Biomechanics. 5: 47-57 (2008).

[29] Srivastava LM, Srivastava V P, Peristaltic transport of a particle-fluid suspension. ASME Journal of Biomechanical Engineering. 111: 157-165 (1989). 
[30] Bhatti MM, Zeeshan A, Ijaz N, Slip effects and endoscopy analysis on blood flow of particlefluid suspension induced by peristaltic wave. J. Mol. Liq., 218: 240-245 (2016).

[31] Kamel, M.H., Eldesoky, I.M., Maher, B.M. and Abumandour, R.M., 2015. Slip effects on peristaltic transport of a particle-fluid suspension in a planar channel. Applied bionics and biomechanics, 2015, 70354 (2015).

[32] Chakraborty, U. S., Biswas, D., \& Paul, M. (2011). Suspension model blood flow through an inclined tube with an axially non-symmetrical stenosis. Korea-Australia Rheology Journal, $23(1), 25$.

[33] T. A. Bég, M.M. Rashidi, O. Anwar Bég and N. Rahimzadeh, Differential transform seminumerical simulation of biofluid-particle suspension flow and heat transfer in non-Darcian porous media, Computer Methods Biomechanics Biomedical Engineering, 16(8):896-907 (2013).

[34] O. Anwar Bég, B. Vasu, T. Sochi and V.R. Prasad, Keller box and smoothed particle hydrodynamic numerical simulation of two-phase transport in blood purification autotransfusion dialysis hybrid device with Stokes and Darcy number effects, Advanced Biotechnology and Bioengineering, 1, 80-100 (2013).

[35] O. Anwar Bég, M.M. Rashidi and N. Rahimzadeh, Tasveer A. Bég and T-K Hung, Homotopy semi-numerical simulation of two-phase thermal haemodynamics in a high permeability blood purification device, J. Mechanics Medicine and Biology, 13, 4, 1350066.1-1350066.26 (2013).

[36] K. Vajravelu, J. Nayfeh, Hydromagnetic flow of a dusty fluid over a stretching sheet, Intern. J Non-Linear Mech, 27, 937-945 (1992).

[37] Hatami, M., Hosseinzadeh, K., Domairry, G., \& Behnamfar, M. T. (2014). Numerical study of MHD two-phase Couette flow analysis for fluid-particle suspension between moving parallel plates. Journal of the Taiwan Institute of Chemical Engineers, 45(5), 2238-2245.

[38] Mahanthesh, B., Gireesha, B.J., Thammanna, G.T., Hayat, T. and Alsaedi, A., 2019. Magnetohydrodynamic squeezing two-phase flow of particulate suspension in a rotating channel with transpiration cooling. Proceedings of the Institution of Mechanical Engineers, Part C: Journal of Mechanical Engineering Science, 233(4), pp.1224-1235.

[39] K. Ramesh, D. Tripathi, O. Anwar Bég, A. Kadir, Slip and Hall current effects on viscoelastic fluid-particle suspension flow in a peristaltic hydromagnetic blood micropump, Iranian Journal of Science and Technology, Transactions of Mechanical Engineering (2018). 
doi.org/10.1007/s40997-018-0230-5 (18 pages)

[40] Ijaz, N., et al. Analytical study on liquid-solid particles interaction in the presence of heat and mass transfer through a wavy channel. Journal of Molecular Liquids 250 (2018): pp 80-87.

[41] Bhatti, M. M., Zeeshan, A., Tripathi, D., \& Ellahi, R. (2018). Thermally developed peristaltic propulsion of magnetic solid particles in biorheological fluids. Indian Journal of Physics, 92(4), 423-430.

[42] Bhatti, M., Sheikholeslami, M., \& Zeeshan, A. (2017). Entropy analysis on electro-kinetically modulated peristaltic propulsion of magnetized nanofluid flow through a microchannel. Entropy, 19(9), 481.

[43] Bhatti, M. M., Zeeshan, A., Ellahi, R., \& Ijaz, N. (2017). Heat and mass transfer of two-phase flow with Electric double layer effects induced due to peristaltic propulsion in the presence of transverse magnetic field. Journal of Molecular Liquids, 230, 237-246.

[44] Eldesoky, I. M., Abdelsalam, S. I., Abumandour, R. M., Kamel, M. H., \& Vafai, K. (2017). Interaction between compressibility and particulate suspension on peristaltically driven flow in planar channel. Applied Mathematics and Mechanics, 38(1), 137-154.

[45] Zeeshan, A., Ijaz, N., \& Bhatti, M. M. (2018). Flow analysis of particulate suspension on an asymmetric peristaltic motion in a curved configuration with heat and mass transfer. Mechanics \& Industry, 19(4), 401.

[46] Khaderi SN, den Toonder JMJ, Onck PR, Microfluidic propulsion by the metachronal beating of magnetic artificial cilia: a numerical analysis. Journal of Fluid Mechanics. 2011; 688: 4465 .

[47] Maraj E, Akbar NS, Nadeem S, Biological analysis of Jeffrey nanofluid in a curved channel with heat dissipation. IEEE Transactions on Nano Bioscience, 204; 13: $431-437$ (2016).

[48] D. Tripathi and O. Anwar Bég, Mathematical modelling of heat transfer effects on swallowing dynamics of viscoelastic flood bolus through the human oesophagus, Int. J. Thermal Sciences, 70, 41-53 (2013).

[49] R. Ellahi et al., Blood flow of Jeffrey fluid in a catherized tapered artery with the suspension of nanoparticles, Physics Letters A, 378, 2973-2980 (2014).

[50] N. S. Akbar, D. Tripathi and O. Anwar Bég, MHD convective heat transfer of nanofluids through a ciliated tube with buoyancy: A study of nano-particle shape effects, Adv. Powder 
Technology, 28 (2) 453-462 (2017).

[51] Abdelsalam, S. I., \& Vafai, K. (2017). Particulate suspension effect on peristaltically induced unsteady pulsatile flow in a narrow artery: blood flow model. Mathematical biosciences, 283, 91-105.

[52] Lakshmi, K. K., Gireesha, B. J., Gorla, R. S., \& Mahanthesh, B. (2016). Effects of diffusionthermo and thermo-diffusion on two-phase boundary layer flow past a stretching sheet with fluid-particle suspension and chemical reaction: A numerical study. Journal of the Nigerian Mathematical Society, 35(1), 66-81.

[53] R.S.R. Gorla, L. Byrd and D. Pratt, Second law analysis for microscale flow and heat transfer, Applied Thermal Engineering, 27 (2007) 1414-1423.

[54] N.S. Akbar and A. W. Butt, Entropy Generation Analysis for Metachronal Beating of Ciliated $\mathrm{Cu}$-water Nanofluid with magnetic field, International Journal of Exergy, 19(1)(2016)41-54

[55] N.S. Akbar and A. W. Butt, Carbon nanotube (CNT)-suspended nanofluid analysis due to metachronal beating of cilia with entropy generation, Journal of the Brazilian Society of Mechanical Sciences and Engineering, 39(6)(2017) 2001-2012 
Greek symbols

\begin{tabular}{|c|l|}
\hline$\tilde{a}$ & Mean width of the channel \\
\hline$B_{0}$ & Magnetic field \\
\hline$\tilde{c}$ & Wave velocity \\
\hline$C$ & Volume fraction density \\
\hline$M$ & Hartmann number \\
\hline$\tilde{P}$ & Pressure in fixed frame \\
\hline$Q$ & Volumetric flow rate \\
\hline Re & Reynolds number \\
\hline $\mathbf{S}$ & Stress tensor \\
\hline$S$ & Drag force \\
\hline$\tilde{t}$ & Time \\
\hline$S_{c}$ & Schmidt number \\
\hline$S_{r}$ & Soret number \\
\hline$B_{r}$ & Brinkman number \\
\hline$\widetilde{U}, \tilde{V}$ & $\begin{array}{l}\text { Velocity components in fixed } \\
\text { frame }\end{array}$ \\
\hline $\bar{X}_{0}$ & Reference position of the cilia \\
\hline$\tilde{X}, \tilde{Y}$ & $\begin{array}{l}\text { Cartesian coordinate axes in fixed } \\
\text { frame }\end{array}$ \\
\hline
\end{tabular}

\begin{tabular}{|c|l|}
\hline$\sigma$ & $\begin{array}{l}\text { Electrical conductivity of Jeffrey } \\
\text { fluid }\end{array}$ \\
\hline$\lambda$ & Wavelength \\
\hline$\mu_{s}$ & Dynamic viscosity of Jeffrey fluid \\
\hline$\phi$ & Amplitude ratio \\
\hline$\rho$ & Fluid density \\
\hline$\varepsilon$ & Measure of cilia length \\
\hline$\lambda_{1}$ & Relaxation time \\
\hline
\end{tabular}




\begin{tabular}{|c|l|}
\hline$\lambda_{2}$ & Retardation time \\
\hline$\gamma$ & Shear rate \\
\hline$\alpha$ & $\begin{array}{l}\text { Eccentricity of the elliptic path of } \\
\text { cilia }\end{array}$ \\
\hline$\beta$ & Wave number \\
\hline
\end{tabular}

Subscripts

\begin{tabular}{|l|l|}
\hline$f$ & Fluid phase \\
\hline$p$ & Particulate phase \\
\hline
\end{tabular}




\section{FIGURES-COMPUTATIONS}

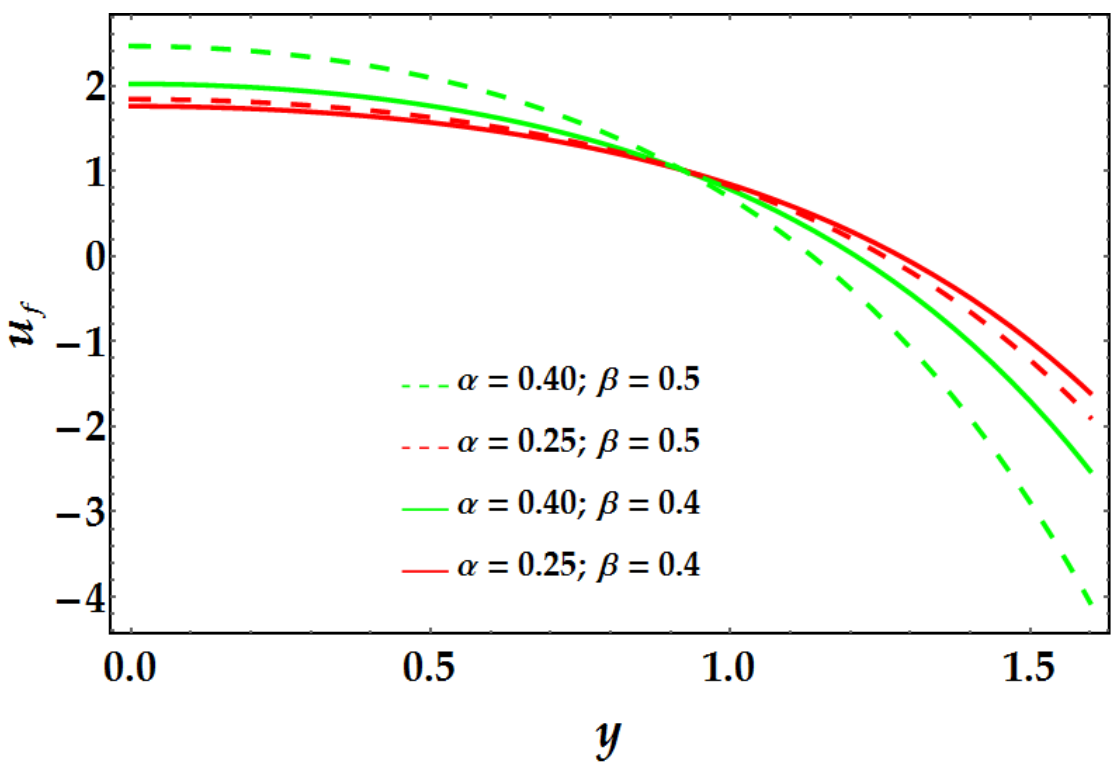

Fig. 2: Velocity profile for various values of $\alpha$ and $\beta$.

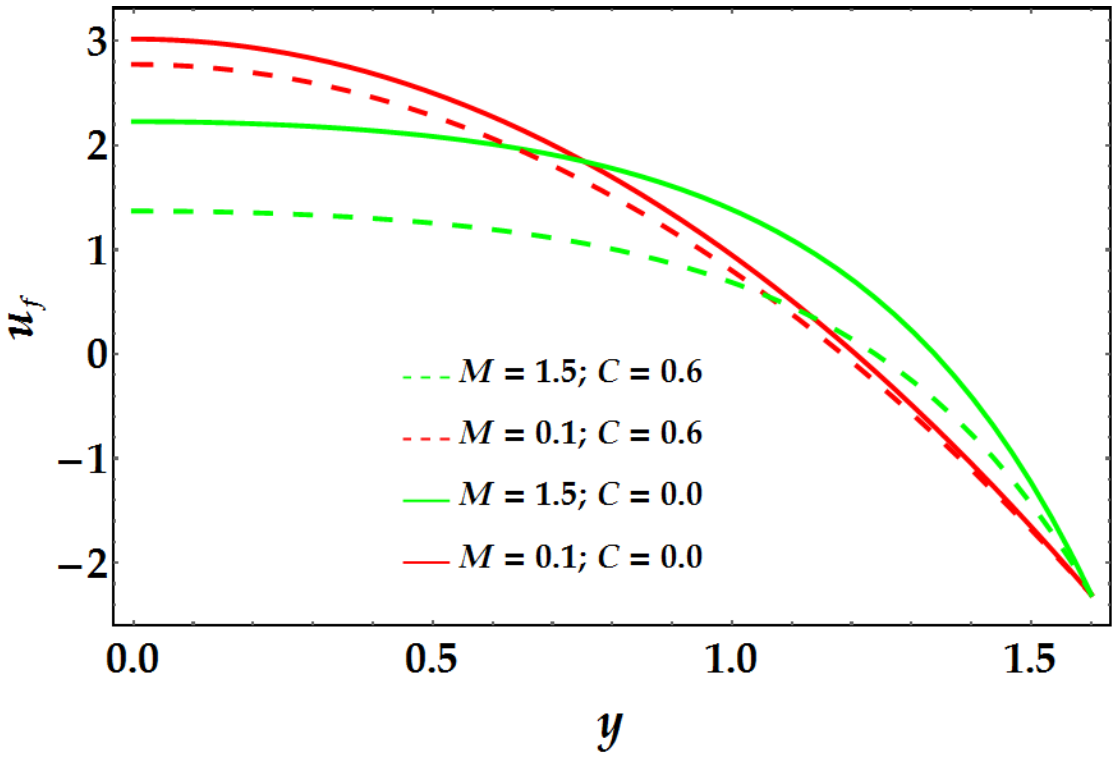

Fig. 3: Velocity profile for various values of $C$ and $M$. 


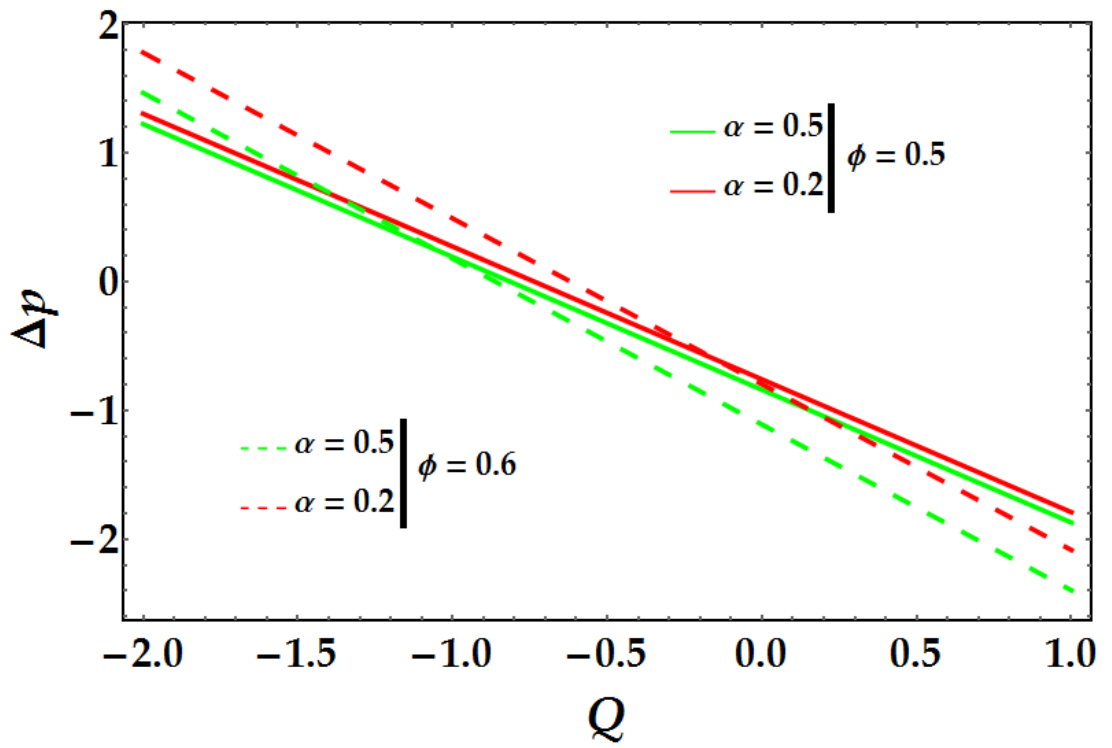

Fig. 4: Pressure rise $v s$ volume flow rate for various values of $\alpha$ and $\phi$.

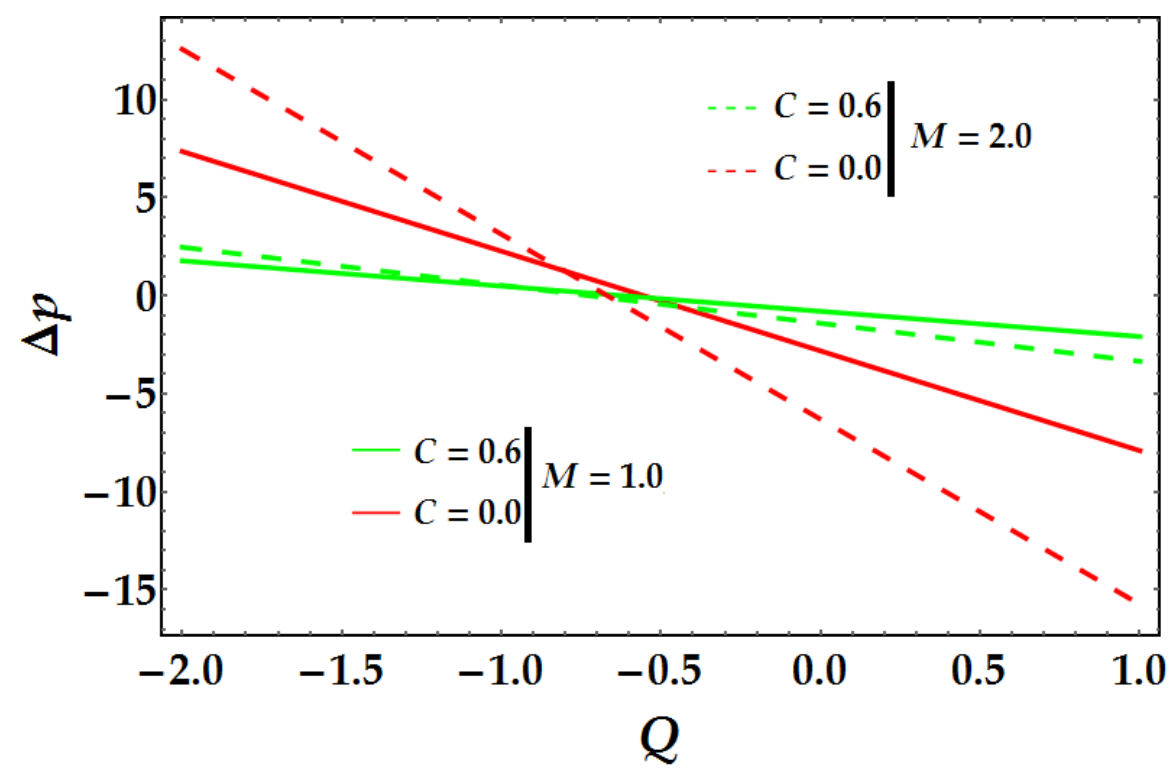

Fig. 5: Pressure rise $v s$ volume flow rate for various values of $C$ and $M$. 

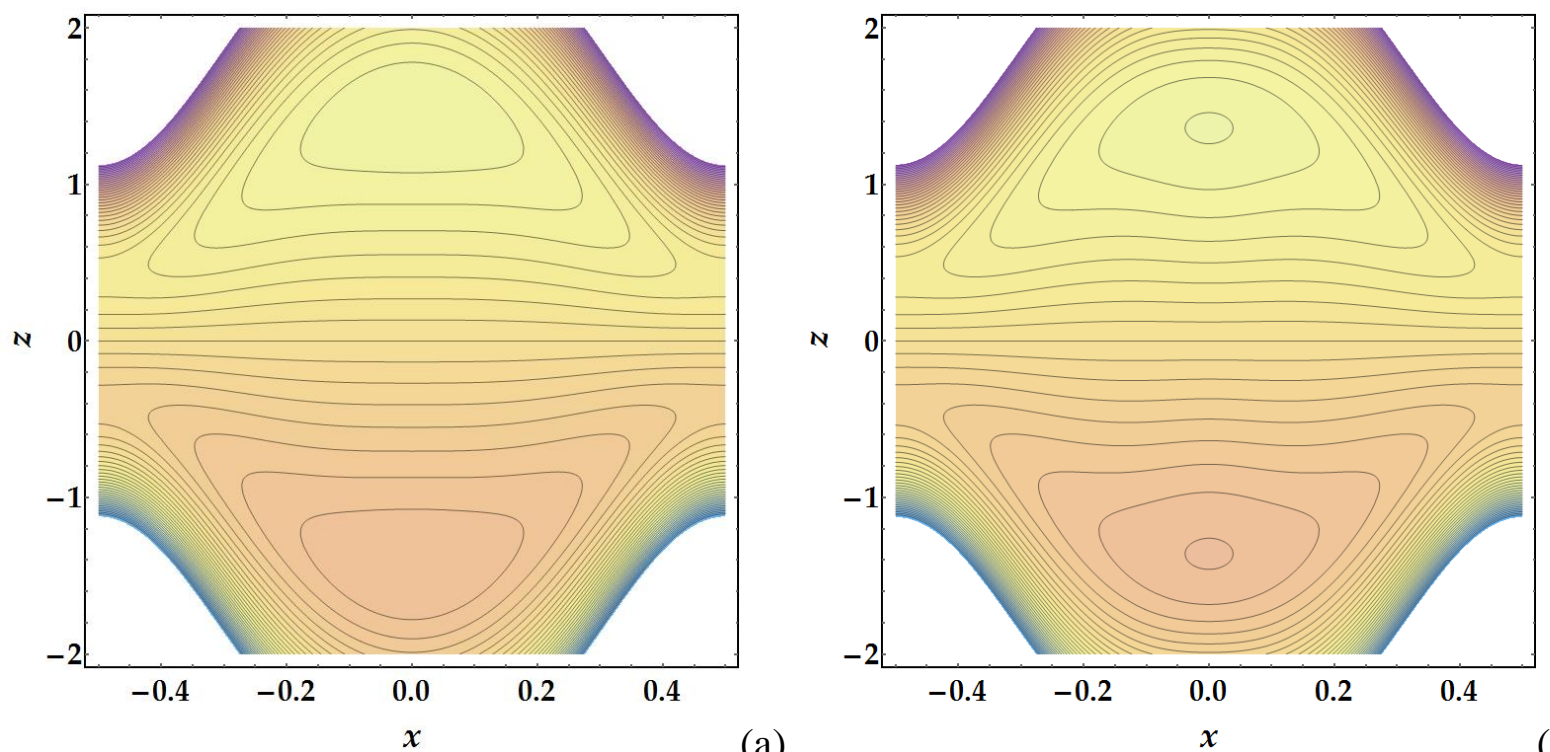

(a)

(b)

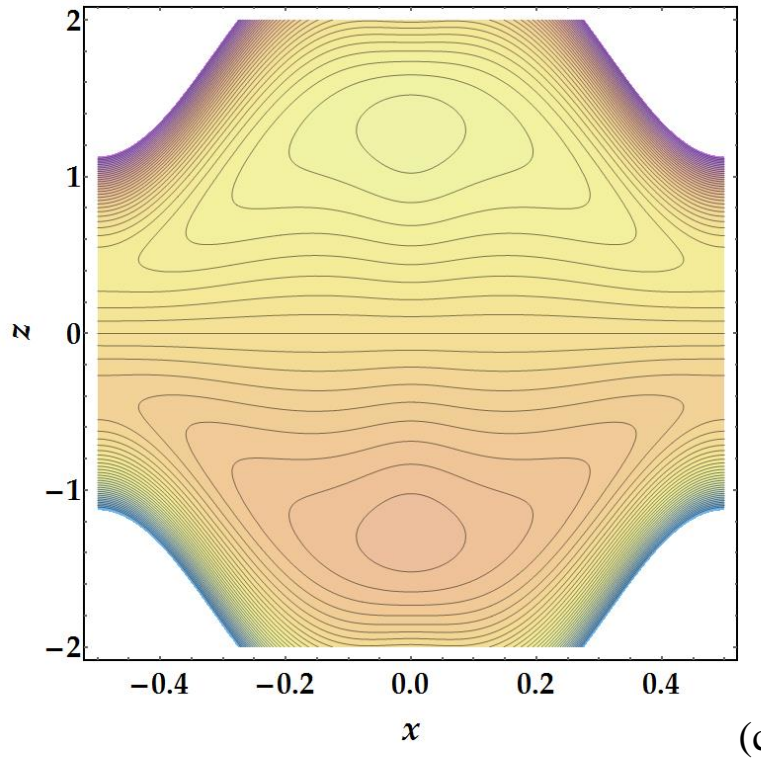

Fig. 6: Stream lines for various values of $\alpha$. $(a) \alpha=0.25 ;(b) \alpha=0.3 ;(c) \alpha=0.4$ 

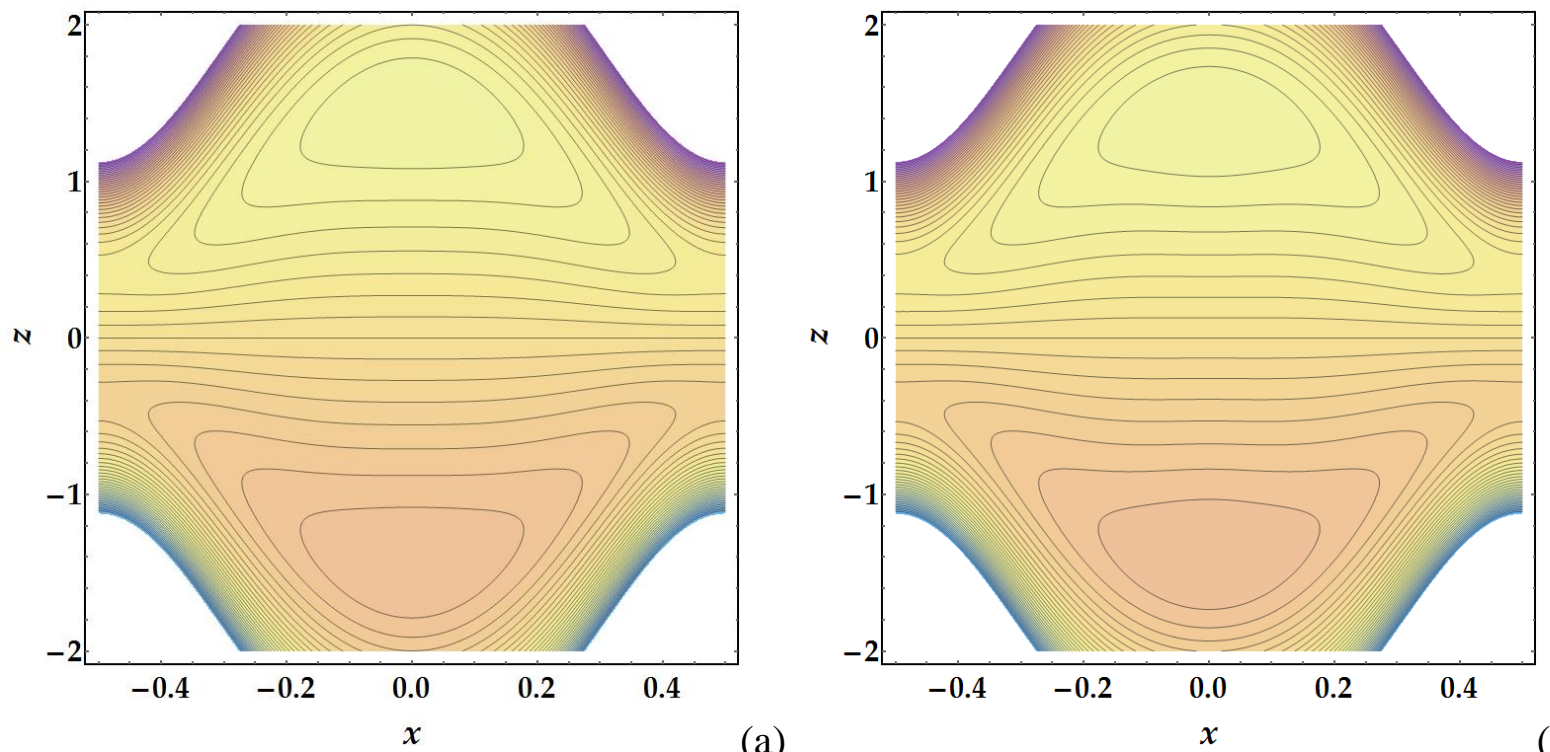

(a)

(b)

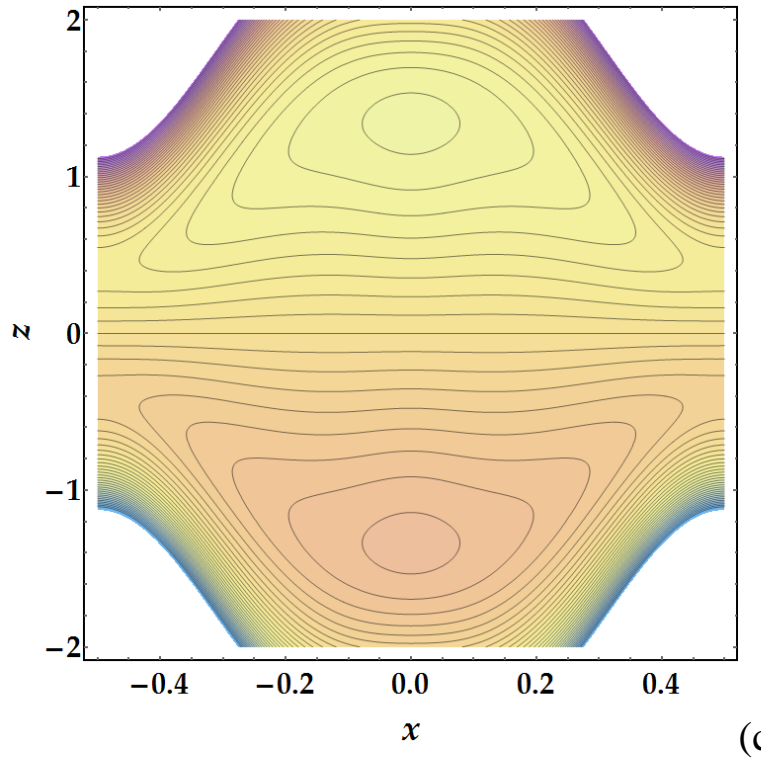

Fig. 7: Stream lines for various values of $\beta$. $(a) \beta=0.4 ;(b) \beta=0.5 ;(c) \beta=0.6$ 

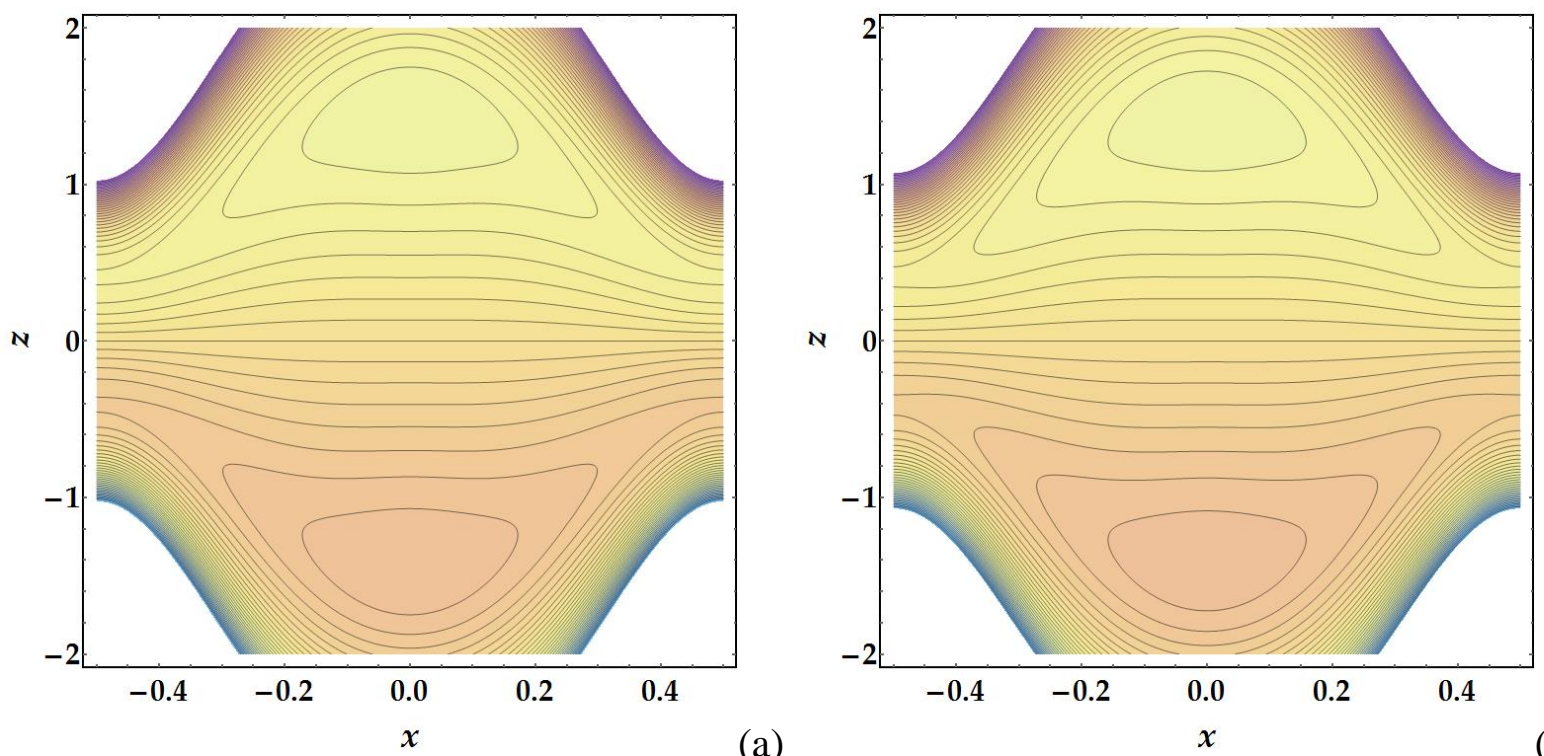

(a)

(b)

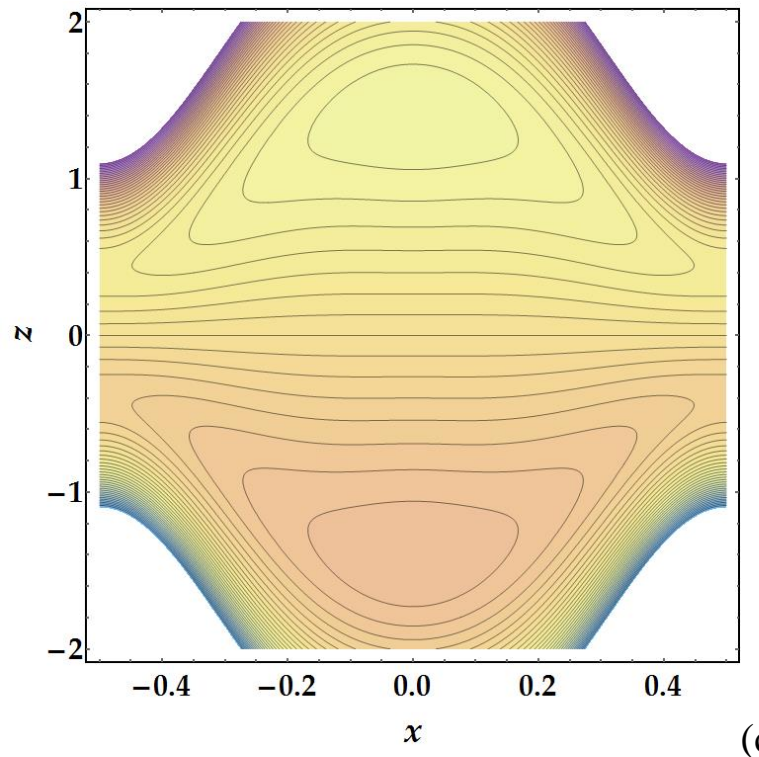

(c)

Fig. 8: Stream lines for various values of $C$. $(a) C=0 ;(b) C=0.15 ;(c) C=0.3$ 

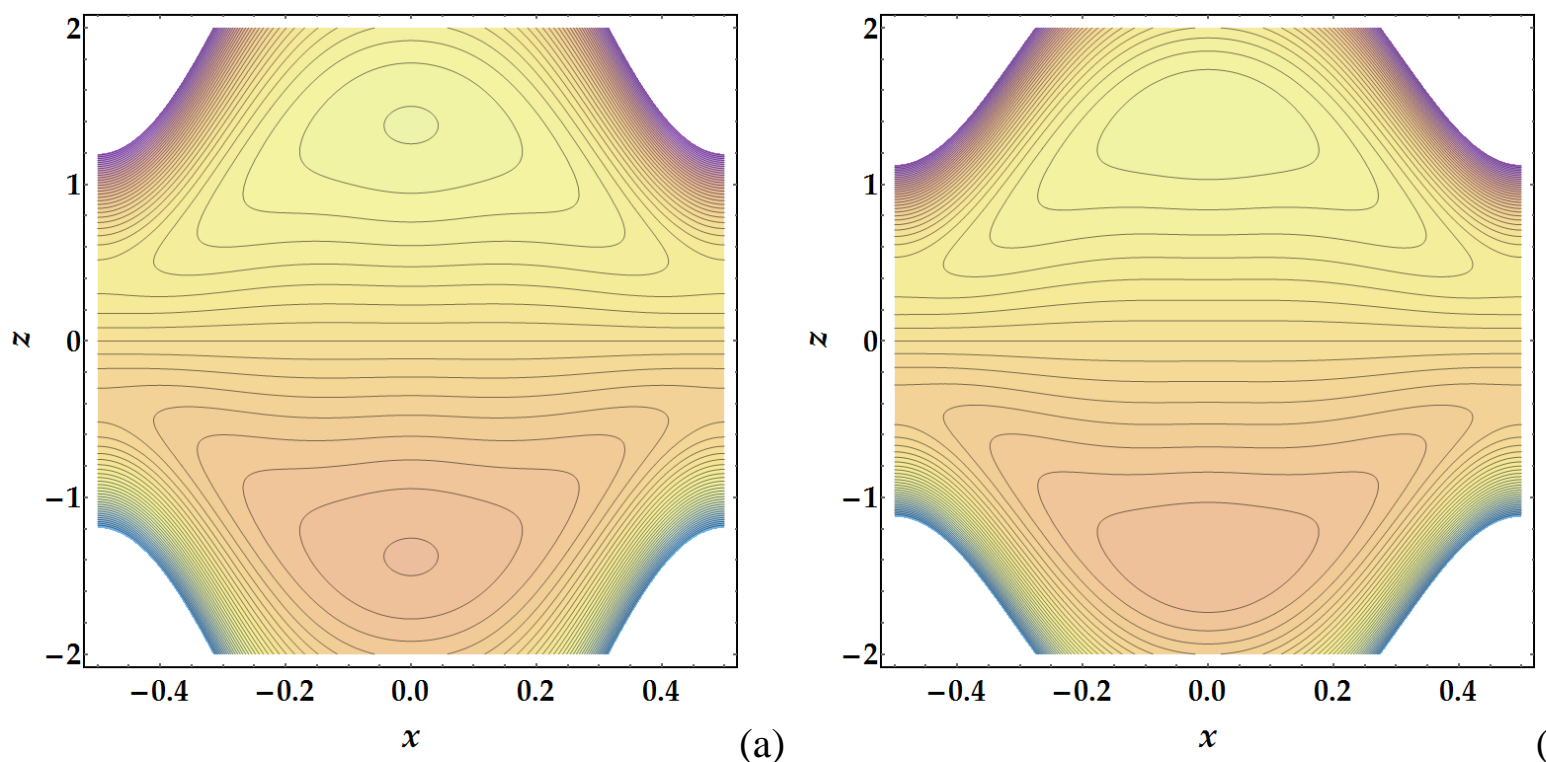

(a)

(b)

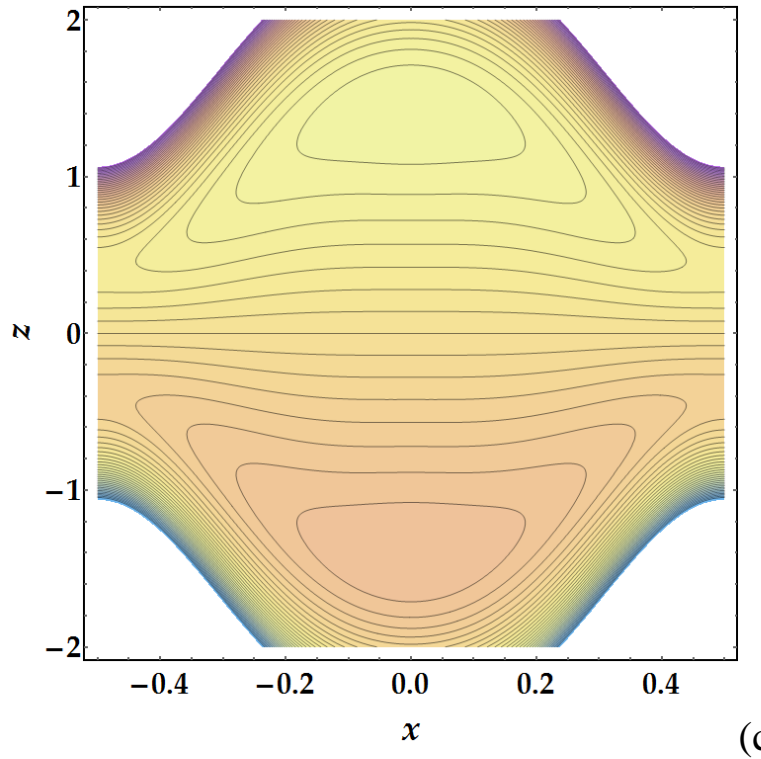

Fig. 9: Stream lines for various values of $M$.(a) $M=0.5$; (b) $M=1.0$ (c) $M=1.5$ 


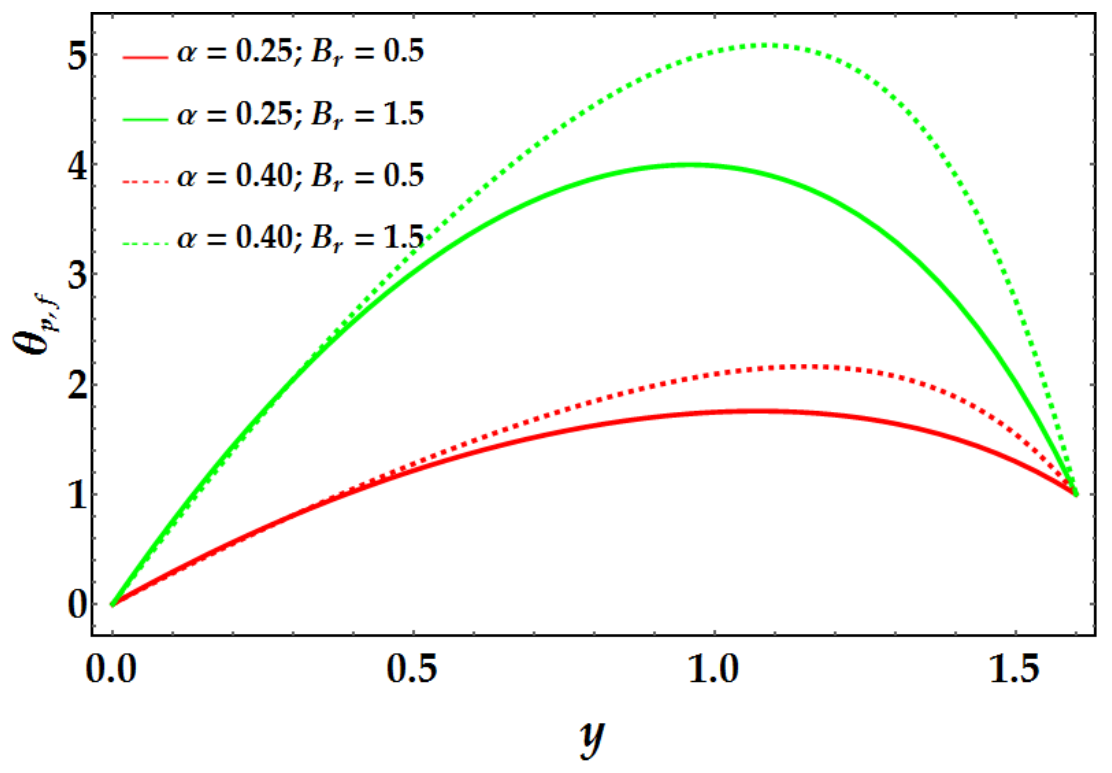

Fig. 10: Temperature profile for various values of $\alpha$ and $B_{r}$.

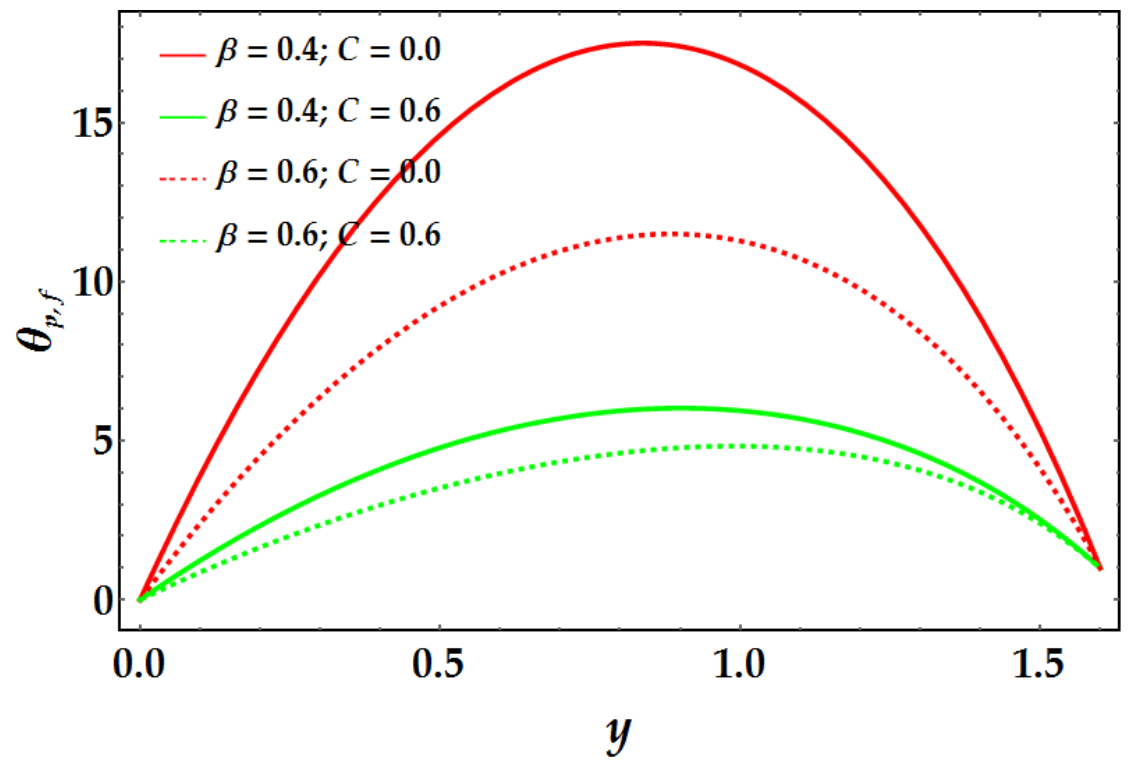

Fig. 11: Temperature profile for various values of $\beta$ and $C$. 


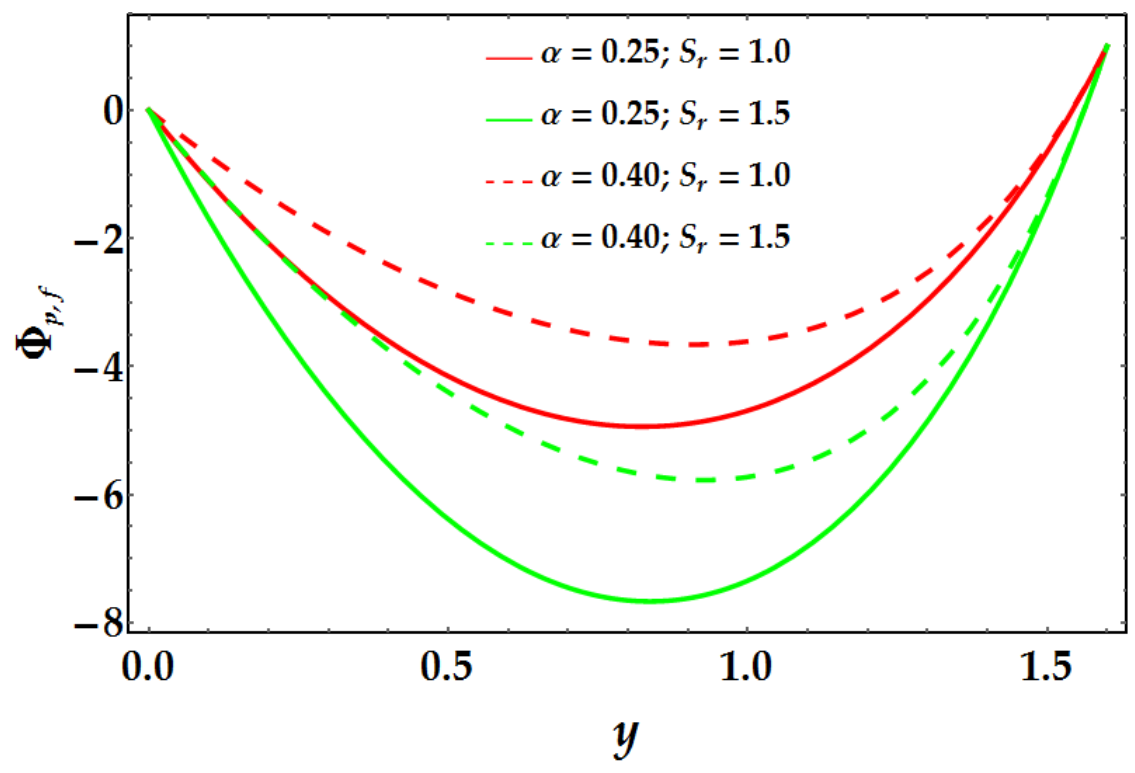

Fig. 12: Concentration profile for various values of $\alpha$ and $S_{r}$.

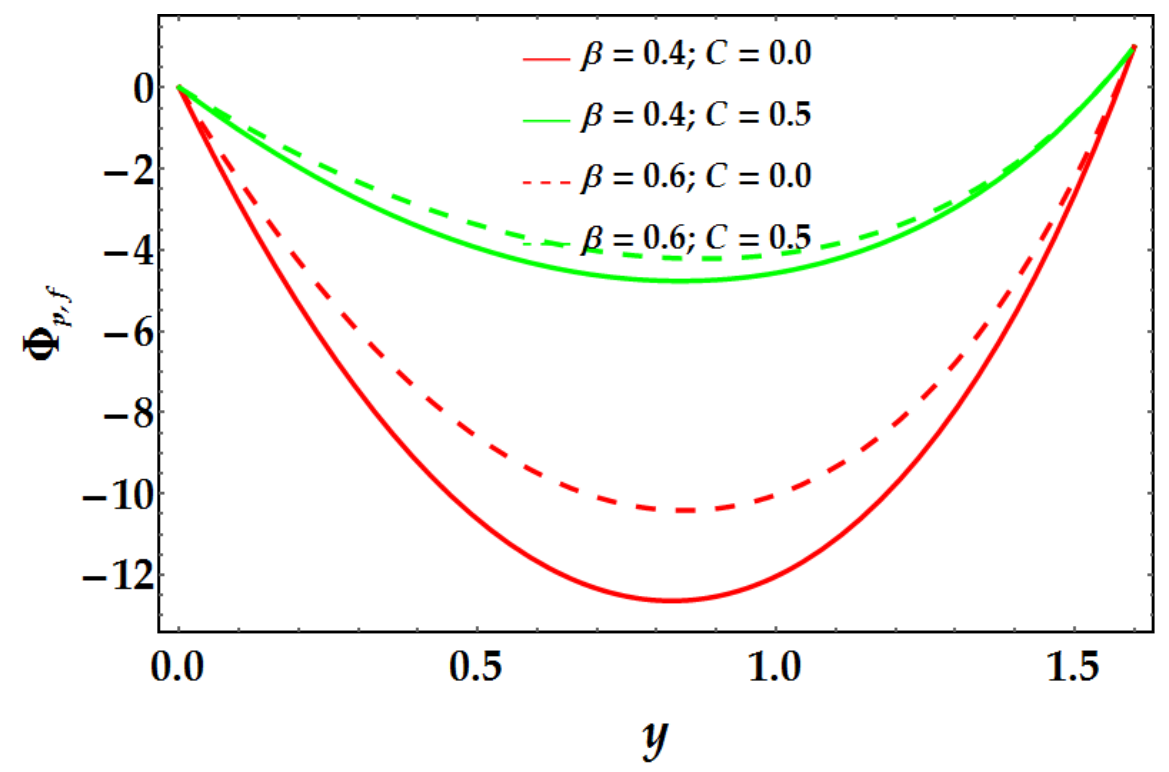

Fig. 13: Concentration profile for various values of $\beta$ and $C$. 


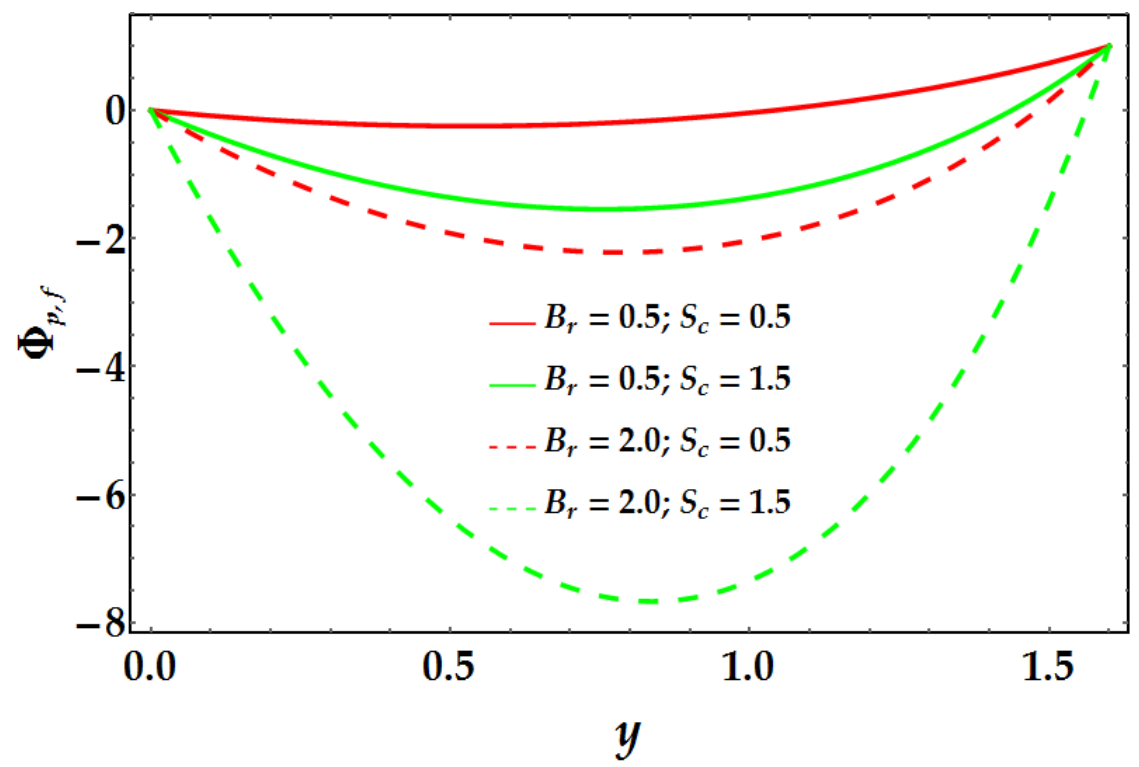

Fig. 14: Concentration profile for various values of $S_{c}$ and $B_{r}$. 